\title{
Output Properties of Zero-Speed Sensors Using FeCoV Wire and NiFe/CoFe Multilayer Thin Film
}

\author{
Yasushi Takemura, Member, IEEE, and T. Yamada
}

\begin{abstract}
Output properties of magnetic sensors generating pulse voltages are described. The sensors principally consisted of double magnetic layers with different coercive forces. Both of thin-film-based material and wire-based material were used for the double layers. When the magnetization of one of the layers was switched by an external magnetic field, a pulse voltage was induced in a pickup-coil wound around the materials. The magnetic sensor using a twisted $\mathrm{FeCoV}$ wire, the conventional material for the Wiegand effect, had the disadvantage of the asymmetric output voltage generated by the alternative magnetic field. It was found that a magnetic wire, whose ends were slightly etched, exhibited symmetric output voltage. The sensor element consisting of a patterned $\mathrm{NiFe} / \mathrm{CoFe}$ multilayer thin film was also studied. Constant output voltage was obtained from this thin-film sensor using an excitation magnetic field at frequencies down to $0.1 \mathrm{~Hz}$.
\end{abstract}

Index Terms-Large Barkhausen effect, magnetic sensor, magnetic thin films, magnetic wires, rotation sensor, velocity sensor, Wiegand effect.

\section{INTRODUCTION}

$\mathbf{T}$ HE MAGNETIZATION switching accompanied with a large Barkhausen jump induces a stable pulse voltage in a pickup coil, which does not depend on the frequency of the exciting magnetic field down to almost $0 \mathrm{~Hz}$. This phenomenon, which is known as the Wiegand effect [1], [2], is utilized for various applications of rotation sensor, speed sensor, and others. A twisted Vicalloy ( $\mathrm{FeCoV}$ ) wire has been studied as the optimum material yielding this effect [3], [4]. Its switching field of 20-30 Oe is relatively high for sensor applications. Another disadvantage in using a wire is the demagnetizing field arising in the wire, which degrades the sensor performance, especially in a short wire. Film-based materials enable a fabrication of a sensor system consisting of a sensor element and an integrated circuit for driving/detecting functions [5]-[7].

We have studied the fabrication and characterization of sensors consisting of both wire and thin-film materials. A sensor using a twisted $\mathrm{FeCoV}$ wire, the conventional material for the Wiegand effect, had the disadvantage of an asymmetric output voltages generated by positive/negative magnetic-field directions [3]. A method of reducing the edge effect by etching both ends of the wire has been proposed [8]. A thin-film-based sensor consisting of $\mathrm{NiFe} / \mathrm{CoFe}$ multilayer thin film deposited on a thermally oxidized $\mathrm{Si}$ substrate was also studied [9].

\footnotetext{
Manuscript received April 30, 2005; revised July 15, 2005. The associate editor coordinating the review of this paper and approving it for publication was Prof. Jian-Ping Wang.

The authors are with the Electrical and Computer Engineering, Yokohama National University, Yokohama 240-8501, Japan (e-mail takemura@ynu.ac.jp).

Digital Object Identifier 10.1109/JSEN.2006.881345
}

TABLE I

COMPARISON OF MAGNETIC SENSORS

\begin{tabular}{|c|c|c|c|}
\hline Sensors & Wiegand sensor & Hall sensor & MR sensor \\
\hline $\begin{array}{c}\text { Detection } \\
\text { principle }\end{array}$ & $\begin{array}{c}\text { Large Barkhausen } \\
\text { jump }\end{array}$ & Hall effect & $\begin{array}{c}\text { Magneto- } \\
\text { resistance effect }\end{array}$ \\
\hline $\begin{array}{c}\text { Major } \\
\text { material }\end{array}$ & $\begin{array}{c}\text { Vicalloy wires } \\
\text { (FeCoV alloy) }\end{array}$ & Semiconductor & $\begin{array}{c}\text { Ferromagnetic } \\
\text { metal }\end{array}$ \\
\hline Power source & Not required & Required & Required \\
\hline Output signal & Constant pulse voltage & Voltage & $\begin{array}{c}\text { Difference of } \\
\text { voltage }\end{array}$ \\
\hline $\begin{array}{c}\text { Temperature } \\
\text { dependence }\end{array}$ & $\begin{array}{c}\text { Independent } \\
\left(-10^{\circ} \mathrm{C} \sim 90^{\circ} \mathrm{C}\right)\end{array}$ & Dependent & Dependent \\
\hline Others & Zero-speed sensor & Low cost & High sensitivity \\
\hline
\end{tabular}

(a)

(b)

(c)

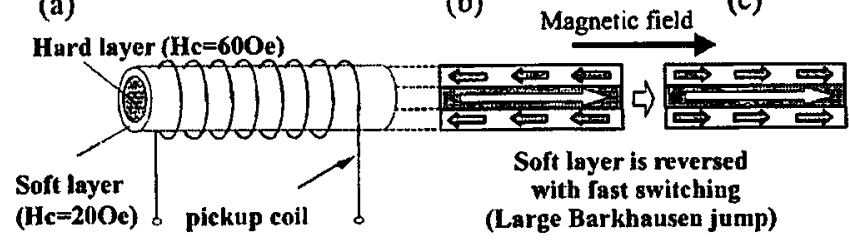

Fig. 1. (a) Double magnetic layers of FeCoV wire. (b) The magnetization configurations of those layers under antiparallel. (c) Parallel alignments.

A sensor element was fabricated by patterning the film by photolithography and wet-etching processes. This thin-filmbased sensor exhibited a constant output voltage for excitation magnetic field at frequencies down to $0.1 \mathrm{~Hz}$.

\section{WIEgAND SENSOR}

Table I shows a comparison of Wiegand sensor, Hall sensor, and magnetoresistive (MR) sensor. The Hall sensor and MR sensor are operated by an electric current through the conducting materials, normally thin films. They require a power source for the current. On the other hand, a Wiegand sensor does not require any power source for an excitation of the sensor element. Fig. 1(a) shows the structure of the twisted $\mathrm{FeCoV}$ wire consisting of double layers of different coercive force. This magnetically compound structure is obtained by twisting the wires. Fig. 1(b) and (c) shows the magnetization configurations of a compound wire under antiparallel and parallel alignments, respectively. In both figures, the magnetization direction of the hard magnetic layer (core) with its coercive 
TABLE II

OUTPUT WAVEForms OF VARIOUS MAgNeTIC SENSORS

\begin{tabular}{|c|c|c|c|}
\hline \multirow{2}{*}{ Sensors } & \multirow{2}{*}{ Detection principle } & \multicolumn{2}{|c|}{ Output waveform } \\
\cline { 3 - 4 } & Hall effect & High speed & Low speed \\
\hline Hall sensor & effect & \\
\hline MR sensor & $\begin{array}{c}\text { Magnetoresistance } \\
\text { Electromagnetic } \\
\text { sensor }\end{array}$ & $\begin{array}{c}\text { Electromagnetic } \\
\text { induction }\end{array}$ & \\
\hline Wiegand sensor & Large Barkhausen jump & & \\
\hline
\end{tabular}

force $H_{\mathrm{c}}=60 \mathrm{Oe}$ is constant. When the magnetization of the soft layer (shell, $H_{\mathrm{c}}=20 \mathrm{Oe}$ ) is switched from the antiparallel to the parallel alignments by the external magnetic field, the pulse voltage is induced to the pickup-coil wound around the wire. Owing to this principle, the sensor element does not require any exciting current. This advantage of "no powersource operation" is another feature of a Wiegand sensor. As the switching speed of the magnetization is almost constant for a wide range of operating temperature up to $90^{\circ} \mathrm{C}$, the output voltage is independent of the temperature. On the other hand, the resistivity of conducting semiconductor materials used for a Hall sensor and ferromagnetic metal for an MR sensor depends much on the temperature. The output signals from these sensors normally depend on the operating temperature.

Table II shows the waveforms of the output voltage for various magnetic sensors. A rotation sensor detecting rotated magnets is assumed for this comparison. The amplitude of the output voltage from a Hall sensor and MR sensor is constant, but the width of the signal depends on the rotation velocity (speed) of the measured objects. The output voltage from a conventional magnetic sensor using electromagnetic induction is proportional to the changing rate of the magnetic field applied to the sensor. Therefore, an electromagnetic sensor is not suitable for detecting slow rotation. A fluxgate magnetometer is a high-sensitive magnetic-field sensor, but the waveform of the output signal also depends on the changing rate of the applied magnetic field.

The magnetization switching of the soft layer of a $\mathrm{FeCoV}$ wire from antiparallel to parallel alignments is fast, since it is accompanied with a large Barkhausen jump. As its switching speed does not depend on the changing rate of the applied magnetic field, the waveform of the output voltage is constant. The Wiegand sensor generates a constant output signal independent of the rotating speed.

Magnetic double layers can be fabricated in a wire or thinfilm-based material. The advantages and disadvantages of a Wiegand sensor consisting of a wire and a thin film are listed in Table III. A twisted FeCoV wire has a uniaxial magnetic anisotropy along the length direction, which is an ideal magnetization configuration for yielding the Wiegand effect. But this uniaxial anisotropy generates demagnetization field at both ends of the wire. It has been proven that a demagnetizing field degrades the frequency dependence on the output properties of a sensor. A relatively large switching field around $20 \mathrm{Oe} \mathrm{might}$
TABLE III

COMPARISON OF WIEGAND SENSOR CONSISTING OF WIRE AND THIN FILM

\begin{tabular}{|c|c|}
\hline Wire material & Thi \\
\hline 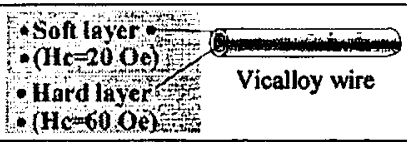 & 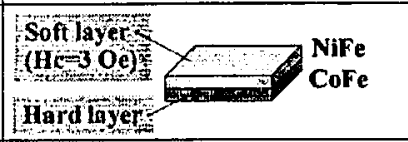 \\
\hline advantage & disadvantage \\
\hline $\begin{array}{l}\text { - Large output voltage( }>20 \mathrm{mV} / \mathrm{tum}) \\
\text {-Ideal uniaxial magnetic anisotropy }\end{array}$ & $\longrightarrow$-Small output voltage (small volum \\
\hline disadvantage & advantage \\
\hline - Limited combinations of materials & $\rightarrow \cdot$ Variety of mate \\
\hline or core/clad structure & $\rightarrow \cdot D$ \\
\hline $\begin{array}{l}\text {-Difficulty in miniaturization or } \\
\text { integration } \\
\text { - Small margin for exciting field } \\
\text { - Large exciting field }(>20 \text { De) }\end{array}$ & $\begin{array}{l}\rightarrow \text { - Suitable for minialurization and } \\
\text { integration } \\
\rightarrow \text { - Wider margin for exciting field } \\
\rightarrow \text { - Low switching field (a few } O e \text { ) }\end{array}$ \\
\hline
\end{tabular}

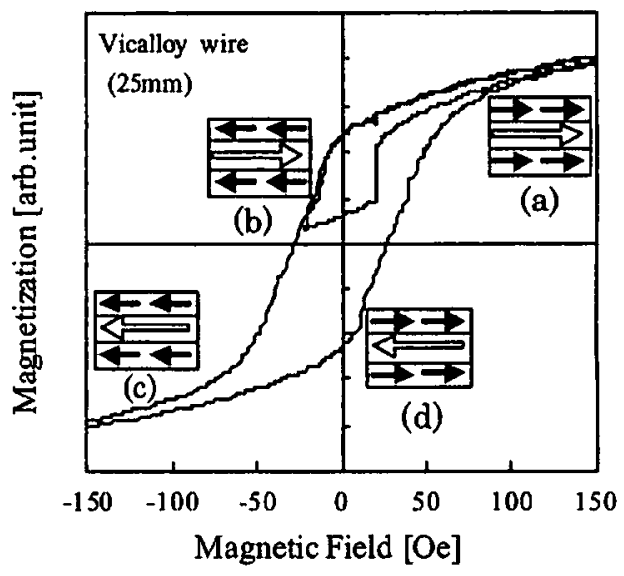

Fig. 2. Magnetization curve of a twisted FeCoV wire

be a problem in a microsized sensor system. On the other hand, the multilayer thin film consisting two layers with different coercive forces is suitable for miniaturizations and fabrications with integrated circuits. A small output voltage owing to the thin thickness and influences of side edges to the magnetization switching are disadvantages.

\section{RESULTS AND DISCUSSION}

\section{A. Wire-Based Wiegand Sensor}

The magnetic wire used in the experiment was a semihard magnetic Vicalloy (40Fe-50Co-10V, wt. \%) wire of $0.25 \mathrm{~mm}$ in diameter. The double-layered magnetic structure was achieved by twisting the wire. This compound wire reveals a uniaxial magnetic anisotropy along the wire axis, and its coercive force is about $20 \mathrm{Oe}$ in the outer shell (soft layer) and $60 \mathrm{Oe}$ in the inner core (hard core). Fig. 2 shows the magnetization curves of a wire measured by a vibrating sample magnetometer (VSM). Both of the major loop and the minor loop are measured. The minor loop, which was recorded by switching the soft layer, while the magnetization of the hard layer was constant. The magnetization reversal of the soft layer from the antiparallel to 


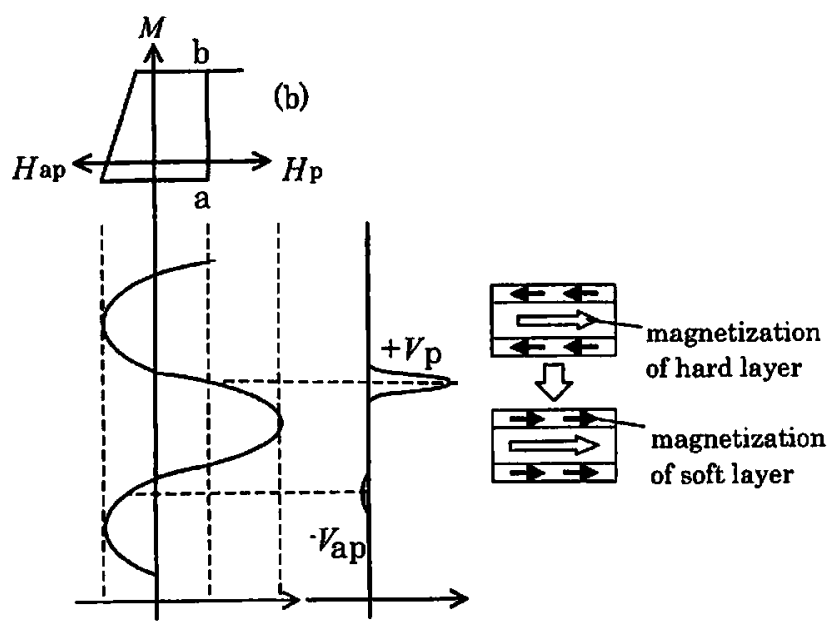

(a)

(c)

Fig. 3. (a) Waveform of the external magnetic field. (b) $M-H$ curve of FeCoV wire. (c) Output pulse voltage.

parallel alignments is steep, which suggests that the switching is accompanied with a large Barkhausen jump.

Fig. 3 shows the $M-H$ curve and corresponding waveforms of exciting magnetic field and output voltages for the wire. When the magnetization of the soft layer is reversed from antiparallel to parallel to that of the hard core (parallel magnetization corresponding to " $a-b$ " in the $M-H$ curve), a sharp pulse voltage indicated by $V_{\mathrm{p}}$ in Fig. 3(c) is induced in the pickup-coil wound around the wire. This magnetic field is defined as parallel field of $H_{\mathrm{p}}$. The amplitude and the half-width of the voltage are typically $2 \mathrm{mV}$ per one turn of the search coil and 10-20 $\mu$ s, respectively. These values are essentially independent of the frequency of the external magnetic field. This is because, the parallel magnetization process is due to the Barkhausen jump, and that, its switching speed is constant. In order to repeat generating the pulse voltage of $V_{p}$, the magnetization of the soft layer should be switched back. During this antiparallel magnetization process by applying $H_{\text {ap }}$, a small voltage indicated $V_{\text {ap }}$ in Fig. 3(c) is also generated.

As the magnetization switching of the soft layer for parallel and antiparallel to the hard core is asymmetric, the optimum condition of the exciting field is also asymmetric. Fig. 4 shows the output voltage $V_{p}$ of the wire obtained by applying the asymmetric alternating field at $70 \mathrm{~Hz}$. The alternating field consisted of $H_{\mathrm{p}}$ and $H_{\mathrm{ap}}$ as shown in Fig. 3(a). The output voltage was measured under various combinations of $H_{\mathrm{p}}$ and $H_{\text {ap }}$. The length of the wire was $30 \mathrm{~mm}$. The voltage per one turn of the pickup coil was indicated. A maximum output voltage was obtained by the asymmetric alternating field of $H_{\text {ap }}=20 \mathrm{Oe}$ and $H_{\mathrm{p}}>50 \mathrm{Oe}$. When $H_{\mathrm{ap}}$ is less than $20 \mathrm{Oe}$, the soft layer does not switched back to the antiparallel configuration. On the other hand, the hard layer is partially switched when $H_{\text {ap }}$ is higher than $20 \mathrm{Oe}$. In both cases of $H_{\text {ap }}$ lower and higher than $20 \mathrm{Oe}$, the ideal configuration of parallel and antiparallel alignments is not achieved, which results in a less output voltage. The optimum condition of this asymmetric exciting field is not suitable for the actual applications using a pair of permanent magnets.

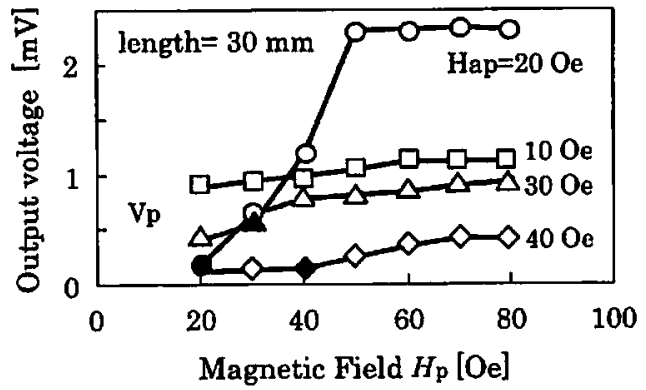

Fig. 4. Output voltage $V_{\mathrm{p}}$ from $\mathrm{FeCoV}$ wire excited various combinations of magnetic fields of parallel and antiparallel directions.

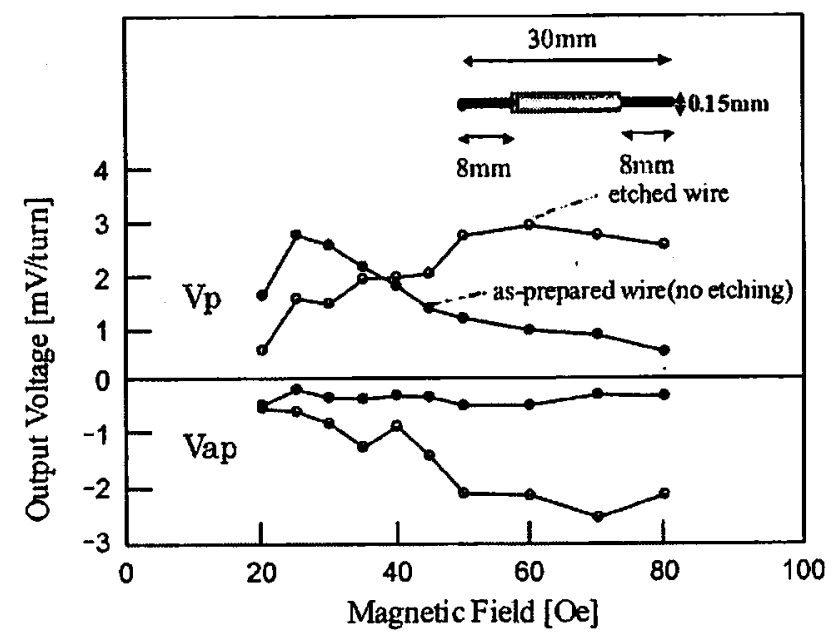

Fig. 5. Output voltage per one tum of pickup coil generated from FeCoV asprepared wire and etched wire. The symmetric ac magnetic field of $H_{\mathrm{p}}=H_{\mathrm{ap}}$ at $70 \mathrm{~Hz}$ was applied.

When a symmetric alternating field is applied to a wire, the output voltages of $V_{\mathrm{p}}$ and $V_{\mathrm{ap}}$, the induced voltages corresponding to parallel and antiparallel magnetization of the soft layer, respectively, are asymmetric. Fig. 5 shows $V_{p}$ and $V_{\text {ap }}$ in positive and negative voltages, respectively, indicated as a function of the magnetic field. The symmetric alternating magnetic field of $H_{\mathrm{p}}=H_{\mathrm{ap}}$ at $70 \mathrm{~Hz}$ was applied. As the antiparallel magnetization reversal was gradual, the negative output voltage of $V_{\text {ap }}$ was small. $V_{\mathrm{p}}$ was larger than $V_{\mathrm{ap}}$, but it decreased with increasing external magnetic field. It was because that the hard layer was also switched with the soft layer. In a practical use, the asymmetric output voltage of $V_{\mathrm{p}}$ and $V_{\mathrm{ap}}$ and the decrease of $V_{\mathrm{p}}$ in the higher exciting field should be avoided. These disadvantages are attributed to the demagnetizing field under the parallel alignment, and also to the magnetostatic coupling between both layers under the antiparallel alignment. In order to reduce these influences at the edge of the wire, the outer layer (soft layer) at both ends was etched by an $\mathrm{FeCl}_{2}$ solution, as shown in the insertion in the figure. This etched wire exhibited almost the same voltages of $V_{\mathrm{p}}$ and $V_{\text {ap }}$. The output voltage was relatively higher than that of the as-prepared wire without etching and did not decrease with increasing the external magnetic field. This etched wire is suitable for practical applications. 


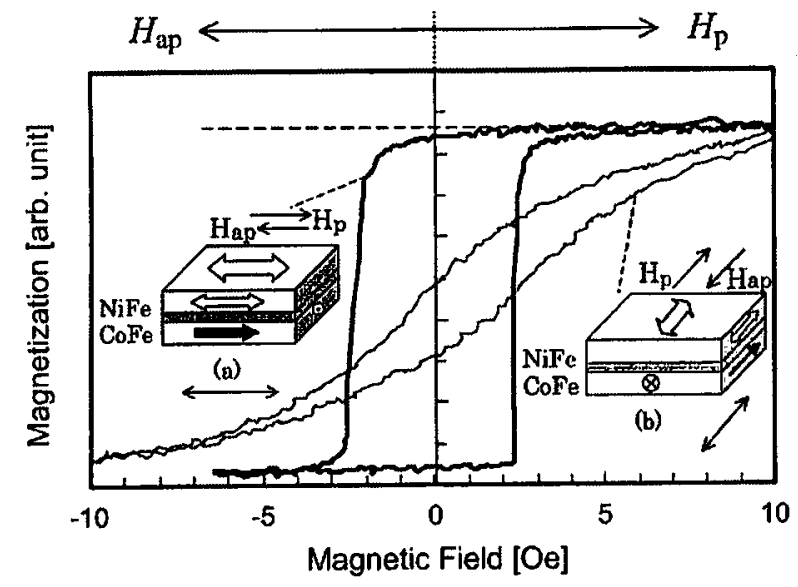

Fig. 6. Minor loops of magnetization curve of $\mathrm{NiFe} / \mathrm{Al}_{2} \mathrm{O}_{3} / \mathrm{CoFe}$ thin film. Bold and thin lines indicate minor loops along (a) easy axis and (b) hard axis of magnetization anisotropy, respectively.

\section{B. Thin-Film-Based Wiegand Sensor}

$\mathrm{NiFe}(300 \mathrm{~nm}) / \mathrm{Al}_{2} \mathrm{O}_{3}(30 \mathrm{~nm}) / \mathrm{CoFe}(150 \mathrm{~nm})$ thin films were prepared on $\mathrm{SiO}_{2} / \mathrm{Si}$ substrate using $\mathrm{RF}$ magnetron sputtering equipment. A uniaxial magnetic anisotropy was induced only in the NiFe layer during the deposition. Magnetization curves of the samples were measured by a VSM. The CoFe layer exhibited an isotropic magnetization along the in-plane direction. A coercive force of the CoFe layer was 50 Oe. Fig. 6 shows minor loops of the magnetization curves measured by switching the magnetization of the NiFe layer under a constant magnetization of the CoFe layer. The magnetic fields of $H_{\mathrm{p}}$ and $H_{\text {ap }}$ indicated in the figure were applied in order to switch the magnetization of NiFe parallel and antiparallel to that of $\mathrm{CoFe}$, respectively. Bold and thin lines indicate the minor loops along the easy and hard axis of magnetic anisotropy of the NiFe Jayer, respectively. Details of the magnetization curves have been discussed in [8]. When the magnetization of $\mathrm{NiFe}$ was reversed to the parallel alignment by applying the magnetic field of $H_{\mathrm{p}}$, the magnetization curve exhibited a steep switching owing to the large Barkhausen effect. This fast switching induces the pulsed voltage in the pickup coil. As indicated in Fig. 6, the magnetization switching to the antiparallel alignment by applying the field of $H_{\text {ap }}$ is gradual, which results in a smaller induced voltage.

When the asymmetric alternating magnetic fields of $H_{\mathrm{p}}=$ $15 \mathrm{Oe}$ and $H_{\mathrm{ap}}=5 \mathrm{Oe}$ were applied to the film, positive and negative output signals of $V_{\mathrm{p}}$ and $V_{\mathrm{ap}}$ were observed, which were associated with parallel and antiparallel magnetization reversals of $\mathrm{NiFe}$, respectively. The amplitude of the output signals is plotted as a function of frequency of the applied magnetic field in Fig. 7. The amplitude of $V_{p}$ was constant at around $12 \mathrm{mV}(1.2 \mu \mathrm{V}$ per turn of the pickup coil) at the frequency range from 1 to $30 \mathrm{~Hz}$, which suggested that the magnetization reversal of $\mathrm{NiFe}$ to the parallel alignment was attributed to a large Barkhausen effect. $V_{\text {ap }}$ was not stably measured at a frequency lower than $20 \mathrm{~Hz} . V_{\mathrm{p}}$ was larger than $V_{\text {ap }}$ at any frequency. Both $V_{\mathrm{p}}$ and $V_{\mathrm{ap}}$ increased with an increasing frequency because of the electromotive force induced in the coil by the alternating magnetic field. The output properties of

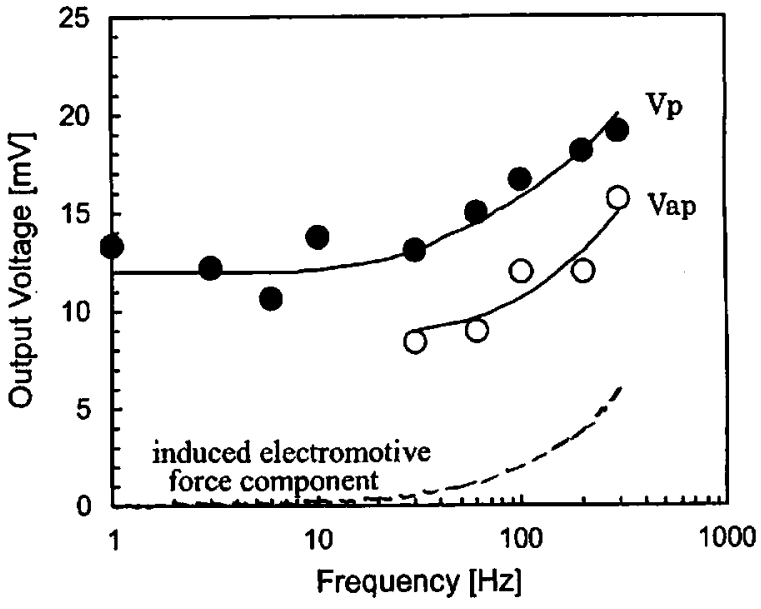

Fig. 7. Frequency dependence of amplitude of output pulse voltage generated from $\mathrm{NiFe} / \mathrm{Al}_{2} \mathrm{O}_{3} / \mathrm{CoFe}$ film.

the sensor using this multilayer thin film in detecting rotated permanent magnets have been reported in [8].

\section{CONClusion}

A Wiegand sensors consisting of wire and thin-film materials were evaluated. The advantages and disadvantages of using a wire and thin films are described. Although a twisted FeCoV wire has been studied as an optimum material for yielding the Wiegand effect, it has the disadvantage of the asymmetric output voltages generated by positive/negative magnetic-field directions. This asymmetric output is caused by the demagnetizing field and magnetostatic coupling at the edge (both ends) of the wire. It was found that this edge effect could be reduced by etching the soft layer at both ends. The etched wire achieved a symmetric output of positive and negative output voltages.

A NiFe/CoFe multilayer thin film deposited on thermally oxidized Si substrate was also studied. A constant output voltage was obtained by using a magnetic excitation field down to a low frequency. The thin-film-based sensor has an advantage of small switching field due to the low coercive force of $\mathrm{NiFe}$ It is also preferable for fabricating with integrated circuits.

\section{ACKNOWLEDGMENT}

The authors would like to thank Prof. A. Matsushita and Dr. S. Abe at Kanagawa University, Kanagawa, Japan, for their valuable discussions, and Mr. Kohno at Nikkoshi Co., Ltd., Japan, for his support on the experiments.

\section{REFERENCES}

[1] J.R. Wiegand and M. Velinsky, "Bistable magnetic device," U.S. Patent 3820090 , Jun. 25, 1974.

[2] P. E. Wigen, "Wiegand wire: New material for magnetic-based devices," Electronics, vol. 48, pp. 100-105, 1975

[3] S. Abe, A. Matsushita, and M. Naoe, "Dependence of large Barkhausen jump on length of a Vicalloy fine wire with torsion stress," IEEE Trans. Magn., vol. 34, no. 4, pp. 1318-1320, Jul. 1998.

[4] Y. Takemura and A. Matsushita, "Frequency dependence of output voltage generated from bundled compound magnetic wires." IEEE Trans. Magn., vol. 37, no. 4, pp. 2862-2864, Jul. 2001 
[5] S. Abe, A. Matsushita, K. Negishi, Y. Baba, and M. Naoe, "Generation of large Barkhausen jump in bilayered thin film," IEEE Trans. Magn., vol. 35 , no. 5, pp. 3634-3636, Sep. 1999.

[6] V. I. Malyutin, "New high-speed planar-type thin film pulse magnetic elements," in INTERMAG Dig., Seattle, WA, 1996. BQ-14.

[7] N. Yano, S. Furukawa, K. Oka, I. Ogasawara, J. Yamasaki, and F. B. Humphrey, "Large Barkhausen discontinuity of Co-Fe-Si-B amorphous films sputtered on polymer substrate," JEEE Trans. Magn., vol. 32, no. 5 , pp. 4532-4534, Sep 1996.

[8] Y. Takemura, M. Nishimoto, T. Aoki, and T. Yamada, "Evaluation of zerospeed sensor using NiFe/CoFe multilayer thin films and twisted FeCoV wires," in Proc. IEEE Sens., 2004, pp. 1090-1093.

[9] Y. Takemura, T. Inoue, M. Nishimoto, and T. Yamada, "Fabrication of zerospeed sensor using weakly coupled NiFe/CoFe multilayer films," IEEE Trans. Magn., vol. 40, no. 4, pp. 2667-2669, Jul. 2004.
Yasushi Takemura (M'99) is an Associate Professor with the Division of Electrical and Computer Engineering, Yokohama National University, Yokohama, Japan.

T. Yamada is a Research Associate with the Division of Electrical and Computer Engineering, Yokohama National University, Yokohama, Japan. 